\title{
Mechanistic Study on Facet-Dependent Deposition of Metal Nanoparticles on Decahedral-Shaped Anatase Titania Photocatalyst Particles
}

\author{
Kenta Kobayashi $^{1}$, Mai Takashima ${ }^{1,2, * \mathbb{C}}$, Mai Takase ${ }^{3}$ and Bunsho Ohtani ${ }^{1,2}$ \\ 1 Graduate School of Environmental Science, Hokkaido University, Sapporo 060-0810, Japan; \\ kobayashi.k@cat.hokudai.ac.jp (K.K.); ohtani@cat.hokudai.ac.jp (B.O.) \\ 2 Institute for Catalysis, Hokkaido University, Sapporo 001-0021, Japan \\ 3 Graduate School of Engineering, Muroran Institute of Technology, Mizumoto-cho, Muroran 050-8585, Japan; \\ mai@mmm.muroran-it.ac.jp \\ * Correspondence: takashima.m@cat.hokudai.ac.jp; Tel.: +81-11-706-9130
}

Received: 11 October 2018; Accepted: 9 November 2018; Published: 13 November 2018

\begin{abstract}
Facet-selective gold or platinum-nanoparticle deposition on decahedral-shaped anatase titania particles (DAPs) exposing $\{001\}$ and $\{101\}$ facets via photodeposition (PD) from metal-complex sources was reexamined using DAPs prepared with gas-phase reaction of titanium (IV) chloride and oxygen by quantitatively evaluating the area deposition density on $\{001\}$ and $\{101\}$ and comparing with the results of deposition from colloidal metal particles in the dark (CDD) or under photoirradiation (CDL). The observed facet selectivity, more or less $\{101\}$ preferable, depended mainly on $\mathrm{pH}$ of the reaction suspensions and was almost non-selective at low $\mathrm{pH}$ regardless of the deposition method, $\mathrm{PD}$ or $\mathrm{CDL}$, and the metal-source materials. Based on the results, the present authors propose that facet selectivity is attributable to surface charges (zeta potential) depending on the kind of facets, $\{001\}$ and $\{101\}$, and $\mathrm{pH}$ of the reaction mixture and that this concept can explain the observed facet selectivity and possibly the reported facet selectivity without taking into account facet-selective reaction of photoexcited electrons and positive holes on $\{101\}$ and $\{001\}$ facets, respectively.
\end{abstract}

Keywords: decahedral-shaped anatase titania particles; $\{001\}$ and $\{101\}$ facets; facet-selective metal photodeposition; $\mathrm{pH}$ dependence; zeta potential; facet-selective reaction

\section{Introduction}

The term "charge separation" is one of the most attractive and convenient terms and/or concepts for researchers in the field of heterogeneous photocatalysis [1,2]. Since charge separation, i.e., spatial separation of a photoexcited electron in the conduction band and a positive hole in the valence band, is a kind of physical process and since the separated charges recombine easily with each other within a very short time period if there is no subsequent chemical reaction consuming those charges, direct observation of the single physical process of charge separation seems practically impossible. Time-resolved pump-probe spectroscopy using a femtosecond laser system has been reported to show such charge separation, i.e., accumulation of trapped photoexcited electrons was completed within a laser pulse and only the decay (disappearance) of those separated charges could be followed. To the best of the authors' knowledge, however, there has been no reported evidence of the primary step of charge separation itself. Then, why has "charge separation" been believed to occur in photocatalyst materials under photoirradiation? One possible reason is speculation assuming a mechanism as an analogy of a photoelectrochemical reaction of semiconductor electrodes, in which there is a space charge layer, i.e., an electric field in a semiconductor being in contact with the electrolyte; for $\mathrm{n}$-type semiconductors such as metal oxides, positive holes and photoexcited electrons are made to migrate 
to the surface and the bulk of an electrode, respectively [2,3]. It should be noted that the depth of the space charge layer depends on the donor density of the electrode material, and it is known that ordinary (non-doped) semiconductor particles such as "white" titanium (IV) oxide particles have a negligible donor density to make the depth larger than the particle size, i.e., there may not be a space charge layer in those particles. Another reason for believing the occurrence of "charge separation" is simple; this concept is very convenient for interpretation of results of photocatalytic reactions; for example, high and low quantum efficiencies or photocatalytic activities have been explained by high and low extents of charge separation without showing direct evidence as described above.

What we observe (or can observe) is the results of chemical reactions that follow physical processes, photoexcitation and possible charge separation. Since the fate of electron-positive hole pairs is limited to an alternative, chemical reaction or recombination, the pairs that are not used to liberate photocatalytic-reaction products must disappear by mutual recombination [4]. Based on a simple kinetic assumption, the overall efficiency of electron-hole utilization is regulated by the ratio of the rate of this alternative; the efficiency must be high and low when the ratio is high and low, respectively. A frequently found misconception in papers on photocatalysis is low efficiency (low activity) being attributed only to faster recombination; slower electron/positive hole transfer to a substrate(s) can reduce efficiency/activity even though recombination occurs at a constant rate. Another misconception involves the recognition of charge recombination as a counter backward process of charge separation, i.e., charge recombination occurs because charges are not spatially separated. Thus, charge separation, a possible physical process just after photoabsorption, has been conveniently used in the interpretation of results of chemical reactions detected in chemical analysis without any support or evidence.

Anyway, if electron-positive hole pairs are created in the bulk of photocatalyst particles, charge separation would be expected to occur only when there is an internal electric field (IEF) in each particle; it seems impossible to separate negative and positive charges without an IEF overcoming the attractive electrostatic force between them. However, as described above, such an IEF does not seem to exist in not heavily (or negligibly) doped semiconducting materials, and even if there is an IEF from the surface to the bulk of a particle, a charge, electron or positive hole, separated to the bulk cannot react with a surface-adsorbed substrate(s). Therefore, when charge separation induced by an IEF is expected, a photocatalyst particle must have (i) two kinds of surfaces with different potentials and (ii) a smoothly changing bulk structure from one surface to the other surface forming a potential slope, i.e., an IEF in the bulk, though such a fine structure, especially providing (ii), seems unrealistic.

Titanium (IV) oxide (titania) is one of the most promising photocatalyst materials and is well known to exhibit high level of photocatalytic activity in various kinds of heterogeneous photocatalytic reactions. Three kinds of crystalline polymorphs of titania (with negligible occurrence of $\mathrm{TiO}_{2}(\mathrm{~B})[5,6]$ ) have been found: Anatase, rutile and brookite as natural minerals in characteristic octahedral, complexed faceted and hexagonal plate-like crystal shapes, respectively. For natural anatase crystals, though they are predominantly octahedral crystals exposing eight equivalent $\{101\}$ facets, decahedral-shaped crystals exposing an additional two $\{001\}$ facets have occasionally been found. On the other hand, when titania particles were prepared in various procedures, octahedral-shaped anatase particles have been rarely found except for reports from the authors' group [7,8], presumably because natural crystals might grow very slowly for satisfying the thermodynamic requirement to expose only the most stable (lowest energy) facets, $\{101\}$. However, detailed analysis revealed that decahedral-shaped anatase particles (DAPs) existed in titania particles, e.g., Evonik (previously Degussa) P25 [9], though selective preparation of DAPs had not been reported before the publications of independent works by Yang and coworkers [10] and the present authors' group [11] using a hydrothermal reaction with a structure-controlling agent (SCA) and using a gas-phase reaction of titanium (IV) chloride and oxygen, respectively.

It has been believed that DAPs exposing two $\{001\}$ and eight $\{101\}$ facets have a high level of photocatalytic activity because photoexcited electrons and positive holes migrate to the $\{101\}$ and $\{001\}$ facets, respectively, resulting in efficient charge separation [12-23]. However, since such "charge 
separation" cannot be observed directly as mentioned above, the reason seems to be just speculation or a hypothesis proposed on the basis of microscopic observations of metal- and metal oxide-deposited DAPs through photocatalytic reduction and oxidation from their precursors on $\{101\}$ and $\{001\}$ facets, respectively [12-20]. There are also reports about facet-selective metal/metal-oxide deposition on the surface of rutile titania and other metal-oxide particles with a polyhedral shape [24-27]. These results have been attributed to facet-selective reduction and oxidation on $\{101\}$ and $\{001\}$ facets, i.e., as far as the authors know, all reports except for one [28] have indicated that photoexcited electrons and positive holes migrate selectively to $\{101\}$ and $\{001\}$ facets, respectively. It seems that this "facet-selective redox (FSR) hypothesis" has become established, and studies are now focusing on the possible mechanism, e.g., facet-dependent band positions [12-14], though the basic assumptions that (i) electron-hole pairs are created in the bulk and then move to the surface and (ii) electrons and holes migrate to different facets depending on the band energies seem inconsistent with (a) observation by femtosecond pump-probe laser spectroscopy of electrons and holes being trapped quickly in the surface states [29] and (b) the fact that charges, electrons and holes, cannot undergo separation and/or directional migration to escape from their electrostatic attraction without an electric field in the bulk of each particle.

However, before discussing the mechanism of FSR, experimental results of photocatalytic metal/metal-oxide deposition leading to the FSR hypothesis may have several problems. For example, (a) the effect of possibly remaining SCAs, used in order to prepare facetted particles, is neglected, (b) the facet-selective deposition has been evaluated only qualitatively using a few scanning electron microscopic images, without showing the number (or volume) ratio of deposits depending on the type of facets and (c) there has been no discussion of the possible migration of metal/metal-oxide deposits after photodeposition.

On the basis of the above-mentioned background, FSR on facetted anatase titania particles was re-examined in this study using (1) DAPs prepared by gas-phase reaction of titanium (IV) chloride and oxygen without using SCAs through (2) quantitative analysis by counting the number of deposited particles in order to evaluate the surface-density ratio of facet-selective deposition via an ordinary photocatalytic reaction and (3) photoassisted deposition from colloidal metal particles; points (1), (2) and (3) were introduced/employed to solve (or suppress) problems (a), (b) and (c), respectively.

\section{Results and Discussion}

\subsection{Decahedral-Shaped Anatase Titania Particles (DAPs) Used in This Study}

The sample particles, DAPs, were prepared by a previously reported procedure, coaxial-flow gas-phase reaction of titanium (IV) chloride and oxygen as schematically shown in Figure 1. One of the features of this DAP sample is its high level of purity, possibly because only titanium, chlorine and oxygen are involved in the preparation system, i.e., no SCAs and even hydrogen sources such as water or organic compounds are included. Although the reason why DAPs exposing only $\{101\}$ and $\{001\}$ facets are selectively prepared has not been clarified yet, it is speculated that DAPs are liberated as a lower surface area/volume ratio only exposing two kinds of facets ( $\{101\}$ and $\{001\})$ as the lowest and appreciably low surface energy [30,31], based on the observation that the observed oblateness (ratio of the short side to the long side of the $\{101\}$ trapezoid ( $b / a$ : see Materials and Methods 3.5.)) was ca. 0.7 and it was not changed even when the preparation conditions were modified (See Materials and Methods). The lowest surface area $\left(\mathrm{nm}^{2}\right)$-volume $\left(\mathrm{nm}^{3}\right)$ ratio $\left(\mathrm{SV}\right.$ ratio; $\left.8.2 \mathrm{~nm}^{-1}\right)$ of ideal decahedral particles is expected to be obtained with oblateness of ca. 0.5 assuming the same surface energy of $\{101\}$ and $\{001\}$ facets. This oblateness of the lowest SV ratio seems smaller than the observed ratio, though a plot of SV ratio as a function of oblateness seems parabolic as shown in Section 3.5 and the SV ratio at 0.7 oblateness, $8.9 \mathrm{~nm}^{-1}$, seems not so high compared with the SV ratio at 0.5 . Although there is still a possibility that the sole by-product of the gas-phase reaction, chlorine, remained on the $\{001\}$ facets even after thorough washing with water and this led to lowering of the $\{001\}$ surface energy and high 
oblateness, it can be stated that the particle shape is not controlled by the surface energy (stability) of each particle, not surface modifiers, and this sample seems suitable for study on FSR behavior. In any case, even if the observed oblateness was 0.7 , more than $70 \%$ of the entire exposed surface area of a DAP was $\{101\}$.

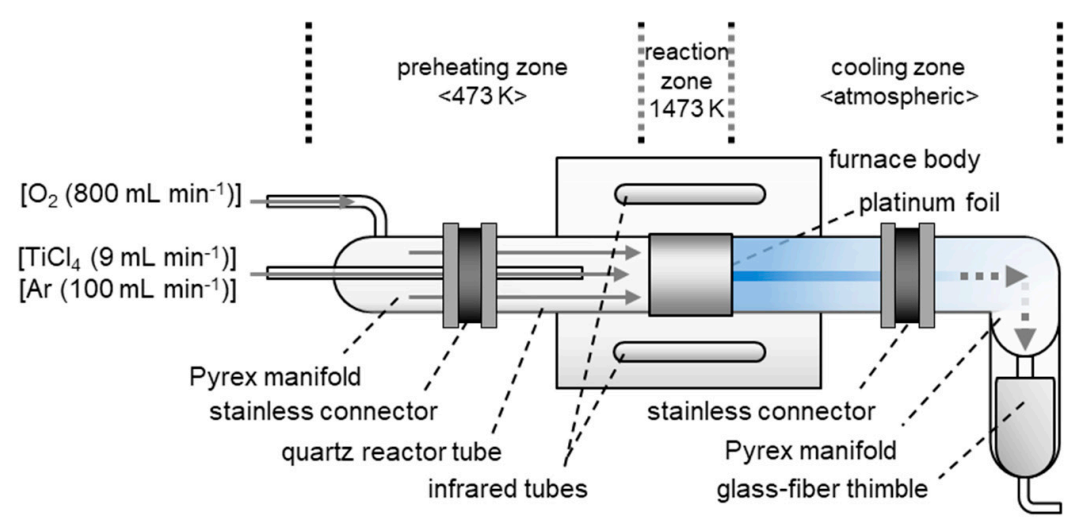

Figure 1. Schematic representation of the DAP synthesis process by coaxial-flow gas-phase reaction of titanium (IV) chloride and oxygen.

Figure 2 shows a representative electron-microscopic image of the sample. In this image, all of the particles have a sharp-edged decahedral shape with sizes of ca. 50-200 nm. Ridges in these observed DAPs looked white due to the "edge effect", i.e., secondary-electron emission occurs preferably at sharp edges. In other words, the DAP samples used in this study have sharp edges. Other images for the samples showed a similar trend except for a few particles with a non-decahedral shape, which might be rutile or non-crystalline titania particles as described below. The crystallite size of anatase evaluated from the XRD patterns with the Scherrer equation was ca. $75 \mathrm{~nm}\left(d_{101}: 71 \mathrm{~nm}, d_{004}: 68 \mathrm{~nm}\right.$ and $d_{200}: 87 \mathrm{~nm}$ ), which was almost the same as the above-mentioned particle size evaluated from SEM images. This rough coincidence suggests that each DAP appearing in SEM images was a single crystal anatase particle.

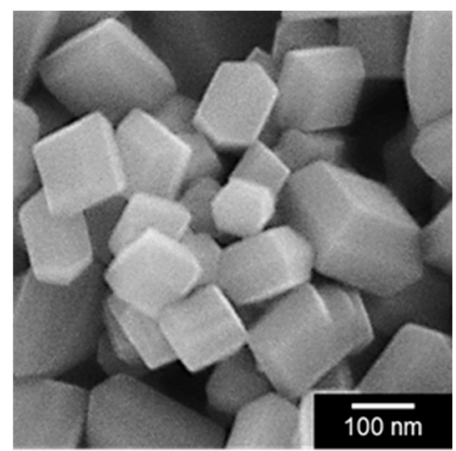

Figure 2. FE-SEM image of DAPs synthesized by coaxial-flow gas-phase reaction.

The crystalline content of the DAP sample used was shown by Rietveld analysis of the diffraction pattern to be $89 \%$ anatase and $4 \%$ rutile with a $7 \%$ non-crystal component.

\subsection{Deposition of Metal Particles on DAPs}

SEM images of gold and platinum-deposited DAPs through photodeposition (PD; (a) and (d)) from precursors and colloid deposition in the dark (CDD; (b) and (e)) and that under photoirradiation $(C D L ;(c)$ and $(f)$ ) are shown in Figure 3. The roughly estimated particle sizes of both gold and platinum deposits, shown in Figure 3, were in the range of 4-12 $\mathrm{nm}$ (Table 1) and no distinct difference in the size depending on the kind of facets was observed. For metal particles photodeposited from their 
precursors, the size was $4-5 \mathrm{~nm}$ regardless of the kind of metals, though the possibility of small $(<1 \mathrm{~nm})$ particle formation could not be excluded due to the resolution of SEM analysis in this study. The average particle size of the original gold colloid was ca. $12 \mathrm{~nm}$ and this was not changed by loading under photoirradiation and in the dark, while the original size of platinum colloid particles was ca. $5 \mathrm{~nm}$. The two-times larger size after deposition in the dark is attributable to possible aggregation of a few platinum particles, though each of them was observed as one particle.
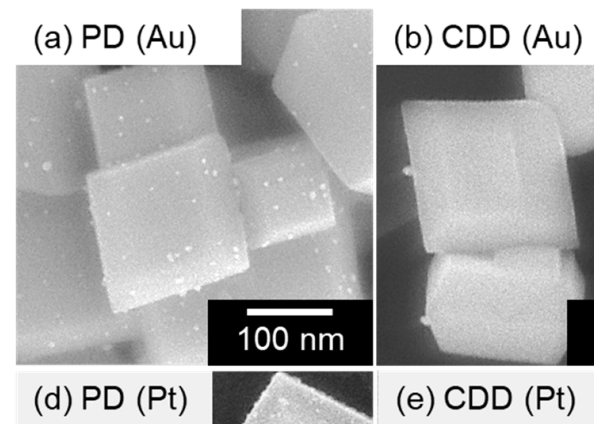

(b) $\operatorname{CDD}(\mathrm{Au})$

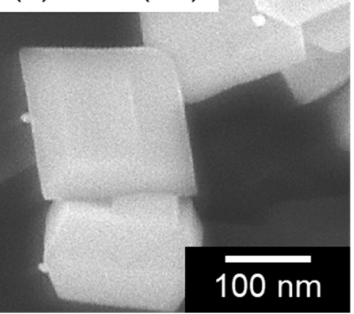

(e) CDD (Pt)

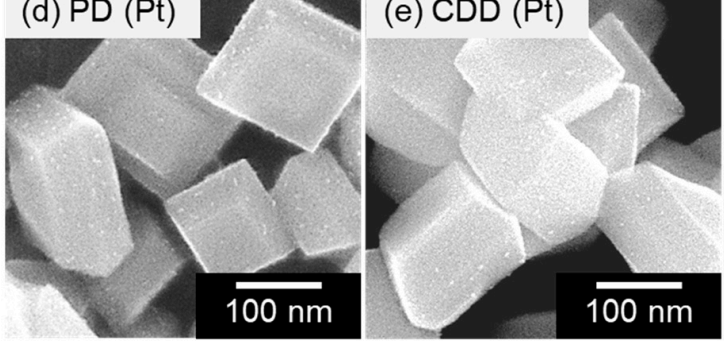

(c) $\mathrm{CDL}(\mathrm{Au})$

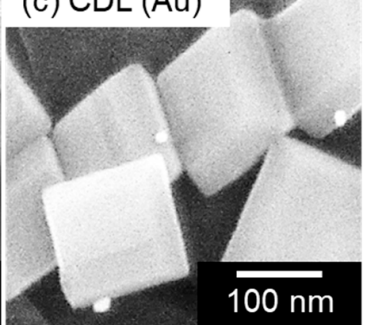

(f) $\mathrm{CDL}(\mathrm{Pt})$

Figure 3. Representative FE-SEM images of DAPs deposited with gold (a-c) and platinum (d-f) by photodeposition (PD) from their precursors ( $\mathrm{HAuCl}_{4}$ and $\mathrm{H}_{2} \mathrm{PtCl}_{6}$, respectively) (a,d), deposition from their colloids in the dark (CDD; $\mathbf{b}, \mathbf{e})$ and under photoirradiation $(\mathrm{CDL} ; \mathbf{c}, \mathbf{f})$.

Table 1. Summary of the results for deposition of metals on DAPs.

\begin{tabular}{|c|c|c|c|c|c|c|c|c|}
\hline Entry & Source & $\begin{array}{c}\text { Amount }^{1} \\
(w t \%)\end{array}$ & Medium & $\mathrm{Size}^{2} / \mathrm{nm}$ & $\mathrm{pH}^{3}$ & $\begin{array}{c}D_{\{001\}}{ }^{4} / 10^{-4} \\
\mathrm{~nm}^{-2}\end{array}$ & $\begin{array}{c}D_{\{101\}} 5 / 10^{-4} \\
n^{-2}\end{array}$ & $S^{6}$ \\
\hline $\mathrm{PD}^{7}(\mathrm{Au})$ & $\mathrm{HAuCl}_{4}$ & 0.5 & $\mathrm{MeOH}^{8}$ & 5 & -9 & 3.9 & 6.4 & 0.61 \\
\hline $\mathrm{CDD}^{10}(\mathrm{Au})$ & Au colloid & 2.0 & water & 12 & -9 & 0.065 & 0.37 & 0.18 \\
\hline $\mathrm{CDL}^{11}(\mathrm{Au})$ & Au colloid & 0.5 & water & 12 & 7.6 & 0.011 & 0.25 & 0.04 \\
\hline $\mathrm{PD}^{7}(\mathrm{Pt})$ & $\mathrm{H}_{2} \mathrm{PtCl}_{6}$ & 0.5 & $\mathrm{MeOH}^{8}$ & 4 & 7.2 & 4.5 & 16.0 & 0.28 \\
\hline $\mathrm{PD}^{7}(\mathrm{Pt} / \mathrm{CA})$ & $\mathrm{H}_{2} \mathrm{PtCl}_{6}$ & 0.5 & $\begin{array}{l}\mathrm{MeOH}^{8} \\
+\mathrm{CA}^{12}\end{array}$ & 5 & 2.5 & 5.1 & 8.2 & 0.62 \\
\hline $\mathrm{CDD}^{10}(\mathrm{Pt})$ & Pt colloid & 2.0 & water & 10 & -9 & 0.12 & 0.48 & 0.24 \\
\hline $\mathrm{CDL}^{11}(\mathrm{Pt})$ & Pt colloid & 0.5 & water & 5 & 7.0 & 0.76 & 16.0 & 0.05 \\
\hline $\mathrm{CDL}^{11}(\mathrm{Pt})$ & Pt colloid & 2.0 & water & 5 & 8.9 & 0.676 & 22.0 & 0.03 \\
\hline $\mathrm{CDL}^{11}(\mathrm{Pt} / \mathrm{CA})$ & Pt colloid & 0.5 & $\begin{array}{c}\text { water } \\
+\mathrm{CA}^{12}\end{array}$ & 4 & 2.4 & 6.6 & 9.0 & 0.73 \\
\hline
\end{tabular}

${ }^{1}$ Amount as metal. ${ }^{2}$ Roughly estimated average size of metal deposits assuming a spherical shape. ${ }^{3}$ Measured after deposition. ${ }^{4}$ Area deposition density of metal deposits for $\{001\}$ facets. ${ }^{5}$ Area deposition density of metal deposits for $\{101\}$ facets. ${ }^{6}$ Facet selectivity $\left(=D_{\{001\}} / D_{\{101\}}\right) .{ }^{7}$ Photodeposition. ${ }^{8} 50 \mathrm{vol} \%$ aqueous methanol. ${ }^{9}$ Not measured. ${ }^{10}$ Colloid deposition in the dark. ${ }^{11}$ Colloid deposition under UV irradiation. ${ }^{12}$ Citric acid $\left(0.1 \mathrm{~mol} \mathrm{~L}^{-1}\right)$.

As a general trend, gold and platinum particles were deposited preferably on $\{101\}$ facets. In order to evaluate the facet selectivity quantitatively, facet selectivity $(s)$ was defined as the ratio of number (not volume) density per unit area, i.e., area density, of metal deposits on $\{001\}\left(D_{\{001\}}\right)$ and $\{101\}$ $\left(D_{\{001\}}\right)$ facets determined by counting more than 100 DAPs in several SEM images for each sample (See Materials and Methods). Since the exposed surface was predominantly $\{101\}$ facets for DAPs used in this study, a comparison without consideration of the surface areas of the two kinds of facets does not seem to make sense. 
For the photodeposited samples, the $s$ values of gold and platinum (in the absence of citric acid) were 0.61 and 0.28 , respectively (Table 1), both of which were below 1, i.e., photodeposition proceeded preferably on $\{101\}$ facets. Although these selectivities were not $0 \%$-or- $100 \%$, the tendency of preferential photodeposition on $\{101\}$ facets seems consistent with previously reported results.

\subsection{Mechanism of Metal Deposition on DAPs and FSR}

Although it has not been discussed so far, the mechanism of photocatalytic metal-particle deposition does not seem to be straightforward since the formation of deposited metal particles composed of a large number of metal atoms requires a process to make metal-metal bonds. At least three steps may be included. (1) The first step is reduction of precursor metal-complex anions $\left(\mathrm{AuCl}_{4}{ }^{-}\right.$ and $\mathrm{PtCl}_{6}{ }^{2-}$ ) adsorbed on the surface of photocatalyst particles by photoexcited electrons along with oxidation of a sacrificial hole scavenger, methanol in the present case, by positive holes. (2a) The next step is migration of low-valent or metal atoms and/or clusters to grow into metal particles (as detected in SEM analysis). It is expected that the larger the size of meal clusters or nanoparticles is, the lower is the possibility of surface migration. (2b) The next step is reduction of metal precursor anions on the surface of formed metal nanoparticles being in contact with titania. Both steps (2a) and $(2 b)$ are probable, and facet-selective deposition is regulated by (i) facet-selective migration of photoexcited electrons and positive holes in step (1), (ii) facet-selective adsorption of metal precursor anions in step $(1 / 2 b)$ and/or (iii) surface migration and facet-selective attachment (fixation) of metal clusters/nanoparticles in steps $(2 a / 2 b)$. At least one of them, if there are no other possible reasons, can lead to a value of $s$ that is different from unity, though previous reports suggested reason (i) without showing evidence that excludes the possibility of (ii) and (iii).

In order to check the possibility of (ii) and (iii), deposition of metal particles on DAPs from gold and platinum colloid solutions was examined in the dark (CDD) and under photoirradiation (CDL). It is well known that colloidal particles prepared using citric acid, which was used in this study, are stabilized by coverage of their surface with citric acid and repulsion with negative charges with carboxylate groups, i.e., the metal-particle surface is negatively charged. For CDD, as shown in Table 1, the area deposition density $(D)$ was lower than that with PD presumably because there seems to be no driving force for colloid particles to settle on the surface (other than interaction with possible protonated hydroxyl groups on the DAP surface) and a higher concentration ( $2 \mathrm{wt} \%)$ of the colloid solution was necessary to count the number of deposited metal-particles. On the other hand, CDL gave a high $D$ compared to that with CDD. Based on the fact that hydrogen and carbon dioxide production was detected and the fact that the particle size of CDL-deposited platinum was almost half of that of CDD-deposited platinum, oxidative decomposition of surface-covering citric acid to expose the bare metal surface proceeded as a counter reaction of hydrogen evolution by photoexcited electrons.

One of the interesting features is that $s$ was less than unity in all cases (PD, CDD and CDL), suggesting that the facet selectivity was governed by the metal-nanoparticle fixation (attachment) process, not the redox process. Another interesting feature is that CDL gave very low s, i.e., high $\{101\}$ selectivity, which was induced by lowering $D_{\{001\}}$ and enhancement of $D_{\{101\}}$ in gold and platinum deposition results, respectively. It seems that these results cannot be interpreted by the conventional mechanism including facet-selective migration of electrons and positive holes if the above-mentioned photocatalytic oxidative decomposition of citric acid on the surface of metal particles induces deposition and no migration of metal particles occurs after the deposition.

\subsection{Influence of $\mathrm{pH}$ on Metal Deposition on DAPs}

Then, what governs the observed facet selectivity? One possible candidate is the surface charge depending on the kind of facets; in all of the deposition methods, PD, CDD and CDL, negatively charged substances, metal-precursor anions or metal colloid particles, are deposited on the surface and such deposition must be influenced by the surface charge of DAPs. 
Figure 4 shows representative SEM images of platinum-deposited DAPs obtained by (a) PD and (b) CDL under acidic conditions. Citric acid was chosen for acidification of the reaction medium since colloidal metal particles contained this acid as a surface stabilizer. As easily seen in those images, platinum particles were deposited both on $\{001\}$ and $\{101\}$ facets and this tendency was evaluated by counting more than 100 DAPs as shown in Table 1 (PD(Pt/CA) and CDL(Pt/CA)); the values of $s$ were greatly increased by the acidification to 0.62 and 0.73 , respectively, which were almost non-selective. On the other hand, a markedly low $s$ value, high $\{101\}$ selectivity, was observed when $\mathrm{pH}$ of the colloidal suspension was basic $(\mathrm{pH}=8.9)$ by using a higher concentration of platinum colloid $(2 \mathrm{wt} \%)$ (Table 1). Thus, $s$ depended strongly on $\mathrm{pH}$ of suspensions in both PD and CDL platinization.
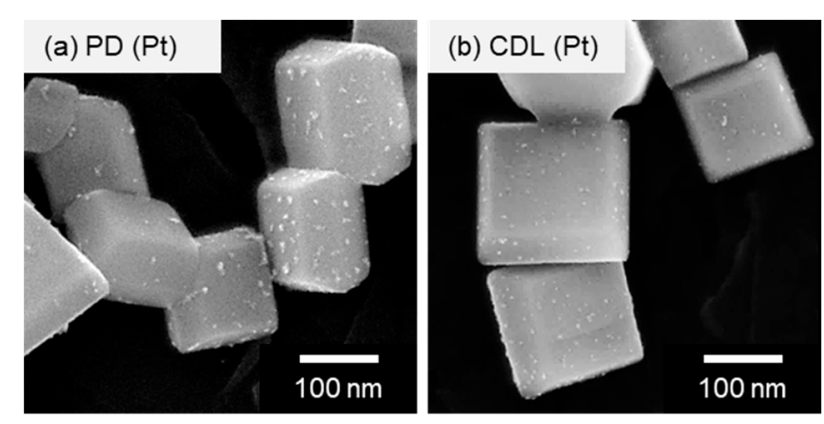

Figure 4. FE-SEM images of metal-deposited DAPs under acidic conditions by: (a) photodeposition; (b) colloid deposition under UV irradiation.

The effect of $\mathrm{pH}$ (acidification) is summarized in Figure 5a. The plots for PD and CDL platinization processes in Figure 5a seem to show resemblance, i.e., their $\mathrm{pH}$ dependence may originate from the same effect. The drastic decrease in the $s$ value along with $\mathrm{pH}$ was caused by the drastic (Note that the $D$ plots are shown in a logarithmic scale.) decrease in $D_{\{001\}}$, while $D_{\{101\}}$ was constant or slightly increased with an increase in $\mathrm{pH}$ raise regardless of the procedure, $\mathrm{PD}$ or CDL, and deposition amount, 0.5 or 2.0 wt $\%$.

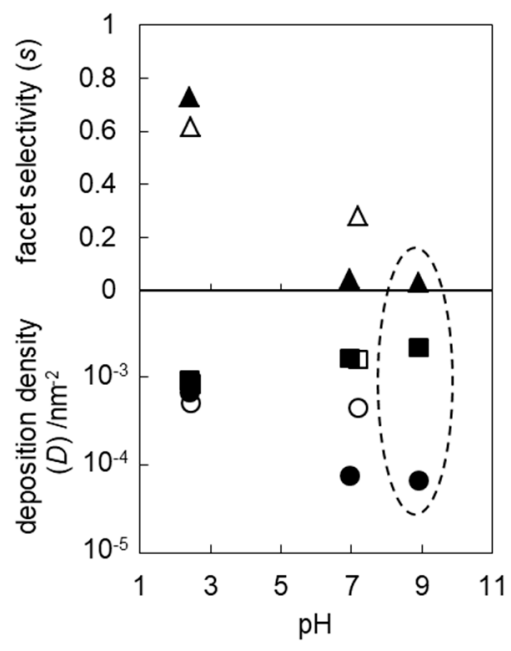

(a)

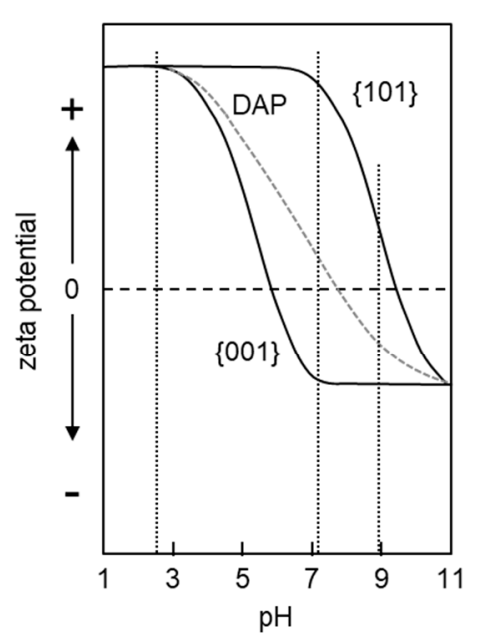

(b)

Figure 5. (a) $\mathrm{pH}$ dependence of facet selectivity (s) and deposition density $(D)$ in PD (open symbols) and CDL (closed symbols) platinum deposition. For deposition-density plots, circles and squares correspond to the density of $\{001\}$ and $\{101\}$ facets, respectively. Plots in the dotted oval reflect samples deposited with a 2 wt \% platinum-colloid solution. (b) Hypothetical pH-dependent zeta-potential curves for $\{101\}$ and $\{001\}$ facets. A dashed line is a rough sketch of actual zeta-potential measurement of a DAP sample giving point of zero charge at ca. 7. It should be noted that the DAP sample used in this zeta-potential change was prepared under slightly different reaction conditions. 
One of the possible reasons for such $\mathrm{pH}$ dependence is $\mathrm{pH}$-dependent and facet-dependent surface charges and, assuming negatively charged species $\mathrm{PtCl}_{6}{ }^{-}$anions or negatively charged platinum colloidal particles are approaching the surface for platinum deposition, changes in surface charge by $\mathrm{pH}$ for $\{101\}$ and $\{001\}$ facets are different. It is well known that there are appreciable amounts of hydroxyl groups on the surfaces of metal-oxide particles, and protonation/deprotonation depending on $\mathrm{pH}$ of a surrounding medium gives protonated $\left(-\mathrm{OH}_{2}{ }^{+}\right)$, neutral $(-\mathrm{OH})$ and deprotonated $\left(-\mathrm{O}^{-}\right)$ forms, resulting in the observed zeta-potential curves; the surface of titania particles is positively charged at low $\mathrm{pH}$, decreased by $\mathrm{pH}$ increase and then negatively charged at high $\mathrm{pH}$.

A hypothesis is that a zeta-potential curve for $\{001\}$ facets is shifted to the lower-pH side from that of $\{101\}$ facets as depicted in Figure $5 b$, being consistent with the actual zeta-potential curve for a DAP sample shown in the figure as a rough sketch. As has been reported for the facet-selective surface charge for bismuth oxybromide [26], different facets with different surface energies may have different surface charges.

The hypothetical zeta-potential curves for $\{101\}$ and $\{001\}$ facets can reasonably interpret the observed $\mathrm{pH}$-dependent $s$ and deposition density $(D)$ shown in Figure $5 \mathrm{a}$ as follows. At low $\mathrm{pH}$, both $\{101\}$ and $\{001\}$ facets are positively charged with protonated surface hydroxyls $\left(-\mathrm{OH}_{2}{ }^{+}\right)$to induce attraction of negatively charged precursor anions/colloidal particles and thereby non facet-selective deposition occurs at $\mathrm{pH}=2.4-2.5$. At neutral $\mathrm{pH}$, the average charge on $\{001\}$ facets is decreased, while $\{101\}$ facets are still positively charged to decrease $s$ at $\mathrm{pH}$ ca. 7. A further increase in $\mathrm{pH}$ leads to negatively charged $\left(-\mathrm{O}^{-}\right)\{001\}$ facets to give negligible facet selectivity at $\mathrm{pH}=8.9$. At higher $\mathrm{pH}$, it is expected that both $\{101\}$ and $\{001\}$ facets are negatively charged, resulting in negligible deposition densities. Under such high $\mathrm{pH}$ conditions, however, large platinum particles were formed and the number of deposits was very small (data not shown), presumably because precursor small metal particles or colloidal particles were aggregated by neutralization of surface negative charges by sodium cations.

Although there has been no experimental evidence for the above-mentioned hypothetical zeta-potential curves due to the lack of a technique for measuring zeta-potentials of each facet on a DAP, the observed facet selectivity in PD and CDL-induced deposition of platinum nanoparticles on DAPs can be consistently explained without taking "charge separation" (FSR) into account. Since there also seems to be no direct evidence for FSR, i.e., speculated only from the position of metal and/or metal oxide deposition, the above-mentioned effect of facet-dependent variation in surface charge may still be a possible reason for the facet-selective (or non-selective) deposition of metals as an alternative of FSR.

\subsection{Influence of Stirring Operation on the Deposition Density of Metal Nanoparticles on DAPS}

When the above-mentioned interaction between the charged surface (facets) and precursor anions or metal nanoparticles is assumed, the possible detachment of the photodeposited metal nanoparticles should be examined. Figure 6 shows the effect of post-irradiation stirring in the dark on Ds for (a) gold and (b) platinum deposition. Although the plots seemed rather scattered, the densities were decreased by post-irradiation stirring except for platinum deposition on $\{101\}$ facets. For gold deposition, the densities on both facets were decreased, but it seemed that gold deposits on $\{001\}$ facets tended to be detached faster than those on $\{101\}$ facets to result in a lower facet-selectivity value. On the other hand, platinum deposits were more stable than gold deposits and the trend of decrease in $D$ was not obvious for both $\{101\}$ and $\{001\}$ facets. The observation of a decrease in the number of deposited metal particles by post-irradiation stirring in the dark indicates that the deposited metal particles can be detached and thereby possibly aggregated to larger particles. 

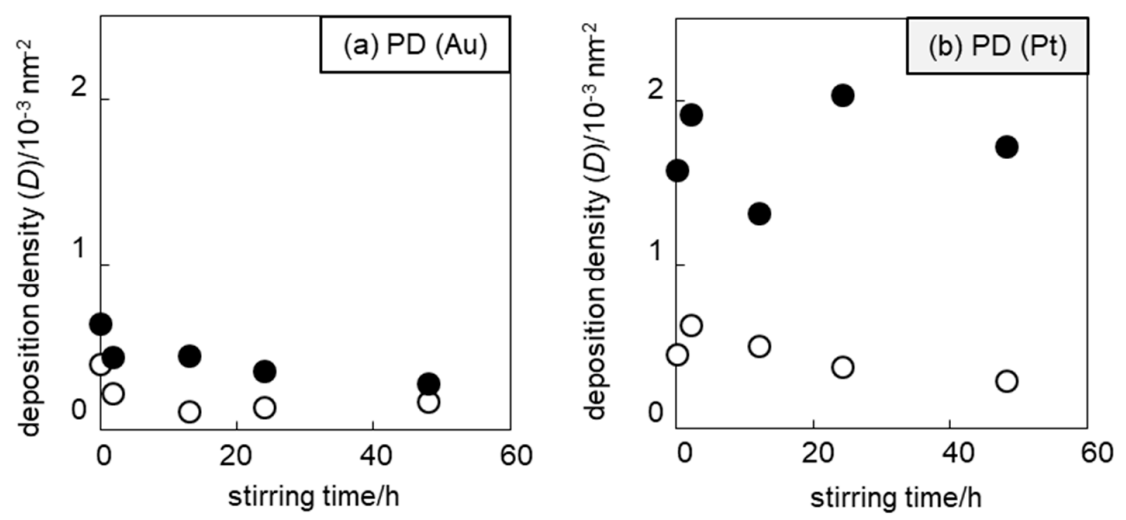

Figure 6. Change in deposition density $(D)$ by post-irradiation stirring of PD processes for (a) gold and

(b) platinum. Open and closed circles correspond to $D$ of $\{001\}$ and $\{101\}$ facets, respectively.

Figure 7 shows the change in the particle size distribution of gold nanoparticles deposited on each facet of DAPs with the time of stirring in the dark after PD. Although only ca. 200 gold particles were counted in each distribution, the number of which seems too small for reliable statistical analysis, the shape of the distribution patterns seemed to change with stirring time. However, a change in the average particle size on each facet was not obvious and thereby the detachment of deposited gold particles proceeded almost homogeneously for both $\{101\}$ and $\{001\}$ facets. Being consistent with the results showing that $\{001\}$ facets tend to release gold nanoparticles faster than do $\{101\}$ facets as shown in Figure 6, the average gold-nanoparticle size on $\{001\}$ facets was a slightly smaller than that on $\{101\}$ facets, i.e., $\{101\}$ facets might be able to keep larger particles than those can be kept on $\{001\}$ facets.

(a) $\{001\}$

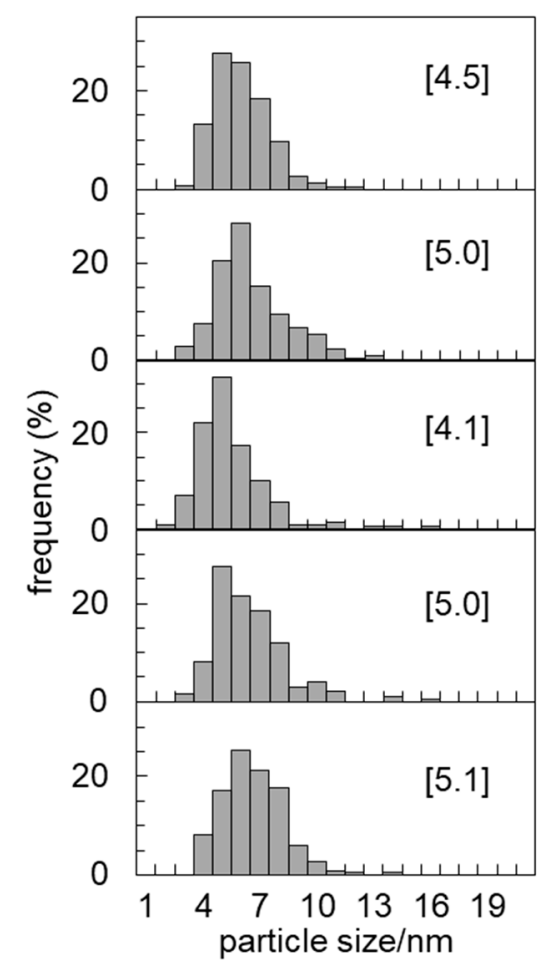

(b) $\{101\}$

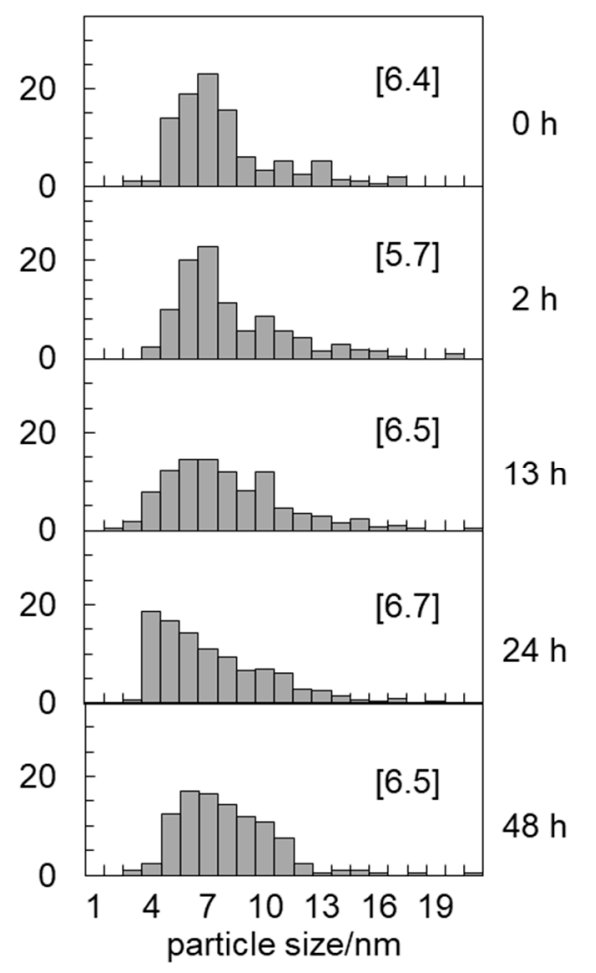

Figure 7. Change in distribution of gold-particle size on (a) $\{001\}$ and (b) $\{101\}$ facets by stirring in the dark for (top) $0 \mathrm{~h}, 2 \mathrm{~h}, 13 \mathrm{~h}, 24 \mathrm{~h}$ and (bottom) $48 \mathrm{~h}$. Frequency was standardized to be $100 \%$ for the 0 -h samples and summation of distribution is proportional to the number of remaining gold nanoparticles. Figures in square brackets show average particle size in the unit of $\mathrm{nm}$. 
Thus, the results suggested that the distribution of metal nanoparticles on $\{001\}$ and $\{101\}$ facets depends on the size and that metal particles that have been deposited can be detached during the process of PD.

\subsection{Photodeposition of Metal Particles on DAPs under No Stirring Conditions}

Since, as described in the preceding section, metal particles deposited in PD processes can be detached from the DAP surface, further experiments using PD without magnetic stirring were performed to reduce the detachment, and the results are summarized in Table 2. In these experiments, DAPs were fixed on a glass plate and irradiated in aqueous methanol containing metal complexes ( $2 \mathrm{wt} \%$ as metal) (See Materials and Methods). Ds were evaluated neglecting DAPs without any metal deposits because unlike PD under the condition of magnetic stirring, only the surface layer of the DAP film absorbs light for metal deposition. Even though such modification in the $D$ evaluation scheme was adopted and a higher concentration of the metal source was used, the actual densities were comparable or even lower than those of PD with magnetic stirring (Table 1). In all of the cases shown in Table 2, $s$ was in the middle range, 0.4-0.6, i.e., ambiguous facet selectivity. Although it is difficult to compare the facet selectivity with that obtained for deposition with magnetic stirring (Table 1) and although facet selectivity for gold deposition without stirring was even decreased, the above-mentioned ambiguous facet selectivity again suggested less probable FSR.

Table 2. Summary of results on PD deposition of metals on DAPs without magnetic stirring.

\begin{tabular}{|c|c|c|c|c|c|c|c|c|}
\hline Entry & Source & $\begin{array}{c}\text { Amount }^{1} \\
(w t \%)\end{array}$ & Medium & $\mathrm{Size}^{2} / \mathrm{nm}$ & $\mathrm{pH}_{3}$ & $\begin{array}{c}D_{\{001\}}{ }^{4} / 10^{-4} \\
\mathrm{~nm}^{-2}\end{array}$ & $\begin{array}{c}D_{\{101\}}{ }^{5 / 10^{-4}} \\
\text { m m }^{-2}\end{array}$ & $S^{6}$ \\
\hline $\mathrm{PD}^{7}(\mathrm{Au})$ & $\mathrm{HAuCl}_{4}$ & 2.0 & $\mathrm{MeOH}^{8}$ & 5 & -9 & 0.28 & 0.75 & 0.38 \\
\hline $\mathrm{PD}^{7}(\mathrm{Pt})$ & $\mathrm{H}_{2} \mathrm{PtCl}_{6}$ & 2.0 & $\mathrm{MeOH}^{8}$ & 4 & 7.2 & 1.3 & 3.3 & 0.39 \\
\hline $\mathrm{PD}^{7}(\mathrm{Pt})$ & {$\left[\mathrm{Pt}\left(\mathrm{NH}_{3}\right)_{4}\right] \mathrm{Cl}_{2}$} & 2.0 & $\mathrm{MeOH}^{8}$ & 5 & 7.4 & 2.0 & 3.5 & 0.58 \\
\hline
\end{tabular}

${ }^{1}$ Amount as metal. ${ }^{2}$ Roughly estimated average size of metal deposits assuming spherical shape. ${ }^{3}$ Measured after deposition. ${ }^{4}$ Area deposition density of metal deposits for $\{001\}$ facets. ${ }^{5}$ Area deposition density of metal deposits for $\{101\}$ facets. ${ }^{6}$ Facet selectivity $\left(=D_{\{001\}} / D_{\{101\}}\right) .{ }^{7}$ Photodeposition. ${ }^{8} 50$ vol \% aqueous methanol. ${ }^{9}$ Not measured.

Another feature seen in Table 2 is that when a cationic precursor, $\left[\mathrm{Pt}\left(\mathrm{NH}_{3}\right)_{4}\right]^{2+}$, was used, the Ds for platinum were comparable or even higher than those for deposition with an anionic precursor, $\mathrm{PtCl}_{6}{ }^{2-}$. This fact suggests that the precursor for deposition which is affected by the surface charge, based on the assumption that $D$ is governed by the surface charge, is small metal particles/clusters, not source metal-complex ions, e.g., $\mathrm{PtCl}_{6}{ }^{2-}$ or $\left[\mathrm{Pt}\left(\mathrm{NH}_{3}\right)_{4}\right]^{2+}$; in the initial stage of $\mathrm{PD}$, small metal particles / clusters are created followed by migration of these precursors with possible particle growth to be fixed on the surface, and the position of deposition in the second step is regulated by surface charges depending on the kind of facets, $\{101\}$ or $\{001\}$. It should be noted that this proposed mechanism does not exclude the possibility of FSR in the first step, and even though facet-selective metal deposition is observed, it seems that this does not prove the occurrence of FSR.

\section{Materials and Methods}

\subsection{Preparation and Characterization of DAP Samples}

DAP samples were prepared by a gas-phase reaction of titanium (IV) chloride ( $\mathrm{TiCl}_{4}$; Wako, Tokyo, Japan) and oxygen $\left(\mathrm{O}_{2}\right)$ using a coaxial-flow gas-phase reactor as reported previously (modified from the original procedure [32]). A brief description of the procedure is as follows. An argon (Ar; $>99.99 \%$; purified by a Shimadzu (Kyoto, Japan) GLC Click-on Triple (hydrocarbon, oxygen and moisture) trap) stream (100 mL min ${ }^{-1}, 453 \mathrm{~K}$ ) containing $1 \mathrm{vol} \% \mathrm{TiCl}_{4}$ (Wako, Tokyo, Japan)(quantitatively introduced by a syringe feeder) vapor and an $\mathrm{O}_{2}$ stream $(>99.5 \%$, dried and purified by a Shimadzu (Kyoto, Japan) GLC Click-on Combi (hydrocarbon and moisture) trap; $800 \mathrm{~mL} \mathrm{~min}^{-1}$ ) are introduced into a quartz 
reactor tube as inside and outside, respectively, coaxial flow and heated from platinum foil $(3.0 \mathrm{~cm})$, wrapped around the quartz reactor tube, the temperature of which is kept at $1473 \mathrm{~K}$ by infrared lamps (Advance Riko VHT-E44, Yokohama., Japan; totally $2 \mathrm{~kW}$ maximum). The preheated outside $\mathrm{O}_{2}$ flow is heated by the $1473-\mathrm{K}$ wrapped platinum foil prior to the inside $\mathrm{TiCl}_{4} / \mathrm{Ar}$ stream and expanded toward the center to react with $\mathrm{TiCl}_{4}\left(\mathrm{TiCl}_{4}+\mathrm{O}_{2} \rightarrow \mathrm{TiO}_{2}+2 \mathrm{Cl}_{2}\right)$. The resultant white titania smoke flows at the center of the reactor tube (see Figure 1 ) and is collected by a glass-fiber filter thimble (Whatman high-purity glass microfiber extraction thimble, $25 \mathrm{~mm} \times 90 \mathrm{~mm}$, Tokyo, Japan). The white product is washed with water five times to remove possibly adsorbed chlorine and then freeze-dried (EYELA FDU-2100, Tokyo, Japan) under vacuum ( $<10 \mathrm{~Pa})$ for $24 \mathrm{~h}$.

The DAP samples were characterized by X-ray diffractometry (XRD) and scanning electron microscopy (SEM) with a Rigaku SmartLab X-ray diffractometer with $\mathrm{CuK}_{\alpha}$ radiation $(40 \mathrm{kV}, 30 \mathrm{~mA}$, Rigaku, Akishima, Japan) and a JEOL JSM-7400F microscope (JEOL, Akishima, Japan), respectively. The details of XRD measurements are as follows: A DAP sample and $20 \mathrm{wt} \%$ nickel oxide (NiO; Wako, Tokyo, Japan) as an internal crystalline standard [33] were mixed thoroughly in an agate mortar, and the XRD pattern of the mixture was recorded with a scanning rate of $1.0^{\circ} \mathrm{min}^{-1}$ and steps of $0.008^{\circ}$ in the $2 \theta$ range of $10-90^{\circ}$. Recorded diffractograms were analyzed using the software PDXL 2 (Version 2.6.1.2, Rigaku, Akishima, Japan) including a RIETAN-FP Rietveld analysis package [33]. Crystallite size, i.e., primary particle size, was estimated by the Scherrer equation with corrected average peak width of anatase 101,004 and 200 peaks at $2 \theta$ of ca. $25.4^{\circ}, 37.8^{\circ}$ and $48.0^{\circ}$, respectively. The detailed conditions and procedure for SEM analysis of the DAP samples are described in the following sections.

\subsection{Photodeposition of Metal Nanoparticles on DAP Samples from Metal Complexes}

In the process of PD of platinum and gold, a 30-mL solution of $50 \mathrm{vol} \%$ aqueous methanol (Wako, Tokyo, Japan) containing hydrogen hexachloroplatinum(IV) $\left(\mathrm{H}_{2} \mathrm{PtCl}_{6}\right.$, Wako, Tokyo, Japan) or aqueous hydrogen tetrachlorogold(III) ( $\mathrm{HAuCl}_{4}$, Wako, Tokyo, Japan) (0.5 wt \% (or $2.0 \mathrm{wt} \%$ ) as metal) was poured in a glass tube containing $0.015 \mathrm{~g}$ of DAP. In some experiments, tetraammineplatinum (II) chloride ( $\left[\mathrm{Pt}\left(\mathrm{NH}_{3}\right)_{4}\right] \mathrm{Cl}_{2}$, Wako, Tokyo, Japan) was used instead of $\mathrm{H}_{2} \mathrm{PtCl}_{6}$, and citric acid (Wako, Tokyo, Japan; $0.1 \mathrm{~mol} \mathrm{~L}^{-1}$ in a suspension) was added to acidify the suspension. The suspension was sonicated to be homogenized, deaerated by argon bubbling, and then irradiated by a 400-W mercury arc (>290 nm; Eiko-sha 400) with vigorous magnetic stirring at $1000 \mathrm{rpm}$. After 15-min irradiation and 2-h irradiation for platinum and gold, respectively, $\mathrm{pH}$ of the suspension was measured using a $\mathrm{pH}$ meter (Horiba $\mathrm{pH}$ meter LAQUA twin, Kyoto, Japan) and the powder was recovered by centrifugation, washed three times with Milli-Q water, and freeze-dried under vacuum $(<10 \mathrm{~Pa})$ for $24 \mathrm{~h}$.

\subsection{Photodeposition of Metal Nanoparticles on DAP Samples from Metal Colloids}

Platinum and gold colloid solutions were prepared following the reported procedures for platinum [34] and gold [35], respectively. For platinum colloid, a 196-mL portion of an aqueous $\mathrm{H}_{2} \mathrm{PtCl}_{6}$ solution $\left(0.30 \mathrm{mmol} \mathrm{L}{ }^{-1}\right)$ was heated to be refluxed by a mantle heater (MS-ES-3, As one, Osaka, Japan) under magnetic stirring. Then $4.0 \mathrm{~mL}$ of aqueous sodium-citrate solution $\left(0.84 \mathrm{~mol} \mathrm{~L}^{-1}\right)$ was added and the reaction mixture was kept boiling for $45 \mathrm{~min}$. After being cooled down rapidly in an ice bath, excess citric acid and inorganic salts in the resultant colloidal solutions were removed by being passed through an ion exchange resin (Organo Amberlite MB-1, Tokyo, Japan)-packed column. For gold colloid, a 202-mL portion of an aqueous $\mathrm{HAuCl}_{4}$ solution $\left(1.0 \mathrm{mmol} \mathrm{L}^{-1}\right)$ was heated to be refluxed, and then $24 \mathrm{~mL}$ of sodium-citrate solution $\left(0.039 \mathrm{~mol} \mathrm{~L}^{-1}\right)$ was added followed by maintenance of reflux for $30 \mathrm{~min}$. The workup procedure was the same as that for the above-mentioned platinum colloid preparation.

Deposition of platinum nanoparticles and deposition of gold nanoparticles under CDL or CDD were performed using $0.015 \mathrm{~g}$ and $0.044 \mathrm{~g}$, respectively, of DAP suspended in a 5.0-mL colloid solution containing the required amount of the metal. The reaction mixture was kept at $298 \mathrm{~K}$ with magnetic 
stirring under photoirradiation, with the same setup as that for PD, or in the dark. The resultant powder was recovered by centrifugation, washed three times with Milli- $Q$ water, and freeze-drying for $24 \mathrm{~h}$.

\subsection{Photodeposition of Metal Nanoparticles on DAP Samples without Agitation}

A $0.20-\mathrm{mL}$ portion of a sonicated DAP suspension $\left(10 \mathrm{mg} \mathrm{mL}^{-1}\right)$ was poured onto a glass plate and dried in vacuum at ambient temperature for $24 \mathrm{~h}$. The DAP-coated glass plate was immersed in $50 \mathrm{vol} \%$ aqueous methanol containing a metal complex, $\mathrm{H}_{2} \mathrm{PtCl}_{6}, \mathrm{HAuCl}_{4}$ or $\left[\mathrm{Pt}\left(\mathrm{NH}_{3}\right)_{4}\right] \mathrm{Cl}_{2}(2 \mathrm{wt} \%$ as metal), and irradiated by a mercury arc at $>290 \mathrm{~nm}$ for $2 \mathrm{~h}$. After metal deposition, the DAP-coated glass plate was dried at room condition without washing procedure.

\subsection{Evaluation of Deposition Densities and Facet Selectivity of Metal Deposition}

The metal nanoparticle-deposited DAPs were analyzed by electron microscopy using a field emission-type scanning electron microscope (FE-SEM; JEOL JSM-7400M, Yokohama, Japan) in a mode of secondary electron image (SEI) with operating conditions of 5.0-10.0-kV electron-acceleration voltage, 10.0- $\mu \mathrm{A}$ current and 3-6-mm working distance. Evaluation of $D$ and $s$ was performed by counting the number, not volume, of metal deposits per unit area in FE-SEM images as follows. First, the number of deposited metal nanoparticles $\left(N_{\{001\}}\right.$ and $\left.N_{\{101\}}\right)$ and total area of deposited facets $\left(S_{\{001\}}\right.$ and $\left.S_{\{101\}}\right)$ were measured using several SEM images for $\{001\}$ and $\{101\}$ facets, respectively. In order to keep statistical reliability and reproducibility, more than 100 metal-deposited DAPs were counted. For the area measurement, the following equations were used to estimate the area of each facet, $S_{\{001\}}$ and $S_{\{101\}}$, with the measured lengths of two ridges of a DAP, long $(a)$ and short $(b)$ sides of a $\{101\}$ trapezoid (Figure 8a). Then the $D$ s on each facet, $D_{\{001\}}$ and $D_{\{101\}}$, were obtained as $N / S$, and $s$ was calculated as $D_{\{001\}} / D_{\{101\}}$. Values of $s$ of more than 1,1 and less than 1 mean $\{001\}$ selective, non selective and $\{101\}$ selective, respectively.

$$
\begin{gathered}
S_{\{001\}}=b^{2} \\
S_{\{101\}}=(a+b) \times h / 2=(a+b) \times(a-b) \times \tan 69.7^{\circ} / 4
\end{gathered}
$$

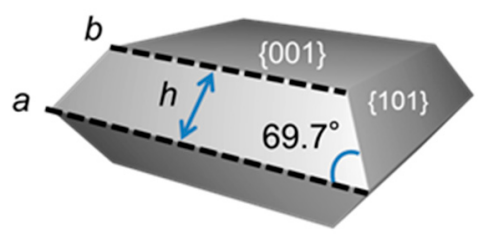

(a)

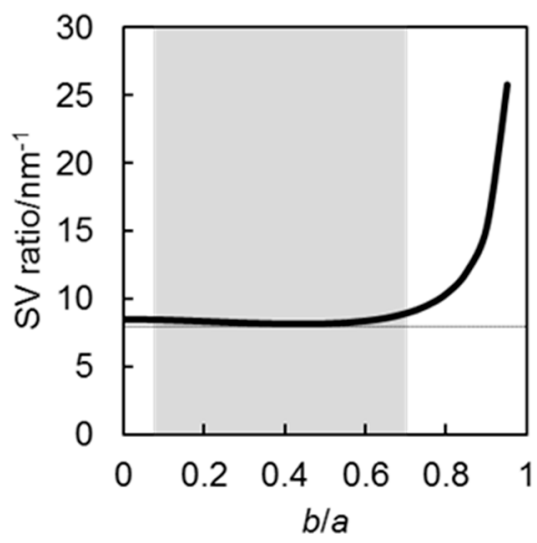

(b)

Figure 8. (a) Assumed dimension of a DAP and (b) surface area-volume (SV) ratio as a function of oblateness, $b / a$, of an ideal $\{101\}$ trapezoid. The grey part corresponds to the oblateness range giving an SV ratio within excess $10 \%$ of the minimum value $\left(8.2 \mathrm{~nm}^{-1}\right)$.

The surface area-volume (SV) ratio, $S_{\text {total }} / V$, of a DAP was calculated using the dimension shown in Figure 8b. 


\section{Conclusions}

As described above, metal-nanoparticle deposition was reexamined using DAPs synthesized by gas-phase reaction of titanium (IV) chloride and oxygen in the absence of a so-called SCA to obtain the following three significant aspects.

One is that the frequently reported almost perfect $(0 \%$-or- $100 \%)$ facet selectivity for photocatalytic deposition, PD, i.e., reductive metal deposition and oxidative metal-oxide deposition on $\{101\}$ and $\{001\}$ facets, respectively, due to FSR could not be observed, at least for the DAP samples used in this study, though there seemed to be a tendency of $\{101\}$-selective deposition. The difference from previously reported results may be due to (i) the conclusion of $0 \%$-or- $100 \%$ selectivity in previous studies by using only one or a few microscopic images matching the FSR concept or (ii) an appreciable difference in the DAP surface structures, e.g., our samples being influenced by the negligibly remaining chlorine or the surfaces of previous samples prepared through liquid-phase processes being covered by an SCA or the others.

The second aspect is that the change in the observed facet selectivity with different reaction conditions was similar to that in deposition of metals, gold and platinum, from CDD or CDL. The FSR concept cannot be applied to CDD since photoexcitation of titania is not induced in this process. Although the detailed mechanism, at least why metal deposition from colloids was enhanced by photoirradiation, has not yet been clarified, the colloid-stabilizing agent citric acid was decomposed along with hydrogen and carbon dioxide evolution and thereby oxidative decomposition/removal of citric acid covering colloidal metal particles may lead to deposition. In such a case, oxidative deposition of metal nanoparticles should be observed on $\{001\}$ facets, not the actually observed $\{101\}$ facets, according to the FSR concept.

The third aspect is $\mathrm{pH}$-dependent change in facet selectivity of platinum-nanoparticle deposition in both PD and CDL processes; $\{101\}$-preferable facet selectivity in neutral and basic $\mathrm{pH}$ conditions became ambiguous at low $\mathrm{pH}$. Based on the assumption that $\{001\}$ facets are more acidic, i.e., easily releasing protons to bear negative surface charges, than are $\{101\}$ facets and that metal nanoparticles are created by the assembly of small atomic or cluster-sized metal precursors, which migrate on the surface in detachment and re-attachment cycles, it is thought that the difference in surface charges depending on the kind of facets and deposition conditions, e.g., $\mathrm{pH}$, accounts for the observed facet selectivity in all of the PD, CDD and CDL processes.

On the basis of these aspects, it can be concluded that the concept of FSR, facet-selective reaction of photoexcited electrons and positive holes, does not seem to be necessary to explain the change in facet selectivity observed in this study and that the results can be consistently interpreted by the possible surface charges depending on the kind of facets, $\{001\}$ and $\{101\}$. Since the procedure and conditions for preparation of DAPs in this study were actually different from those used in previous studies, there might be another mechanism for the reported facet selectivity. Furthermore, the results of this study, non $0 \%$-or- $100 \%$ facet selectivity, do not suggest that only $0 \%$-or- $100 \%$ selectivity results were chosen in previous studies since possibly different surface structures of DAPs used in previous studies might have led to $0 \%$-or- $100 \%$ selectivity. However, the present authors propose here that the above-mentioned surface charge-dependent deposition of platinum and gold nanoparticles can explain the results shown in this article and may be expanded to the previously reported results.

Author Contributions: All authors: draft preparation, writing and editing. K.K.: performing all of the experiments and data analyses. M.T. (Mai Takase) and B.O.: design, maintenance and instruction of the gas-phase reaction for preparation of DAPs. M.T. (Mai Takashima): project management and maintenance of FE-SEM. B.O.: project leading and fund acquisition.

Funding: This research was partly supported by a Grant-in-Aid for Scientific Research (A) (Grant Numbers: 15H0220106 and 18H0392308) from Japan Society for the Promotion of Science (JSPS).

Acknowledgments: FE-SEM analyses of samples were carried out using a JEOL JSM-7400F electron microscope at Global Facility Center, Creative Research Institution, Hokkaido University Technical assistance and support for construction and maintenance of the gas-phase reactor for DAP synthesis and the other instrumental setups by the Technical Division of Institute for Catalysis, Hokkaido University are acknowledged. 
Conflicts of Interest: The authors declare no conflict of interest.

\section{References}

1. Ohtani, B. Preparing Articles on Photocatalysis-Beyond the Illusions, Misconceptions, and Speculation. Chem. Lett. 2008, 37, 216-229. [CrossRef]

2. Ohtani, B. Revisiting the fundamental physical chemistry in heterogeneous photocatalysis: Its thermodynamics and kinetics. Phys. Chem. Chem. Phys. 2014, 16, 1788-1797. [CrossRef] [PubMed]

3. Ohtani, B. Photocatalysis A to Z-What we know and what we do not know in a scientific sense. J. Photochem. Photobiol. C Photochem. Rev. 2010, 11, 157-178. [CrossRef]

4. Ohtani, B. Titania Photocatalysis beyond Recombination: A Critical Review. Catalysts 2013, 3, 942-953. [CrossRef]

5. Banfield, J.F.; Veblen, D.R.; Smith, D.J. The identification of naturally occurring $\mathrm{TiO}_{2}(\mathrm{~B})$ by structure determination using high-resolution electron microscopy, image simulation, and distance-least-squares refinement. Am. Mineralogist 1991, 76, 343-353.

6. Banfield, J.F.; Bischoff, B.L.; Anderson, M.A. $\mathrm{TiO}_{2}$ accessory minerals: Coarsening, and transformation kinetics in pure and doped synthetic nanocrystalline materials. Chem. Geol. 1993, 110, 211-231. [CrossRef]

7. Amano, F.; Yasumoto, T.; Prieto-Mahaney, O.-O.; Uchida, S.; Shibayama, T.; Ohtani, B. Photocatalytic activity of octahedral single-crystalline mesoparticles of anatase titanium (IV) oxide. Chem. Comm. 2009, 2311-2313. [CrossRef] [PubMed]

8. Amano, F.; Yasumoto, T.; Prieto-Mahaney, O.-O.; Uchida, S.; Shibayama, T.; Terada, Y.; Ohtani, B. Highly Active Titania Photocatalyst Particles of Controlled Crystal Phase, Size, and Polyhedral Shapes. Top. Catal. 2010, 53, 455-461. [CrossRef]

9. Ohtani, B.; Iwai, K.; Nishimoto, S.-I.; Sato, S. Role of Platinum Deposits on Titanium (IV) Oxide Particles: Structural and Kinetic Analyses of Photocatalytic Reaction in Aqueous Alcohol and Amino Acid Solutions. J. Phys. Chem. B 1997, 101, 3349-3359. [CrossRef]

10. Yang, H.G.; Sun, C.H.; Qiao, S.Z.; Zou, J.; Liu, G.; Smith, S.C.; Cheng, H.M.; Lu, G.Q. Anatase TiO 2 single crystals with a large percentage of reactive facets. Nature 2008, 453, 638. [CrossRef] [PubMed]

11. Amano, F.; Prieto-Mahaney, O.-O.; Terada, Y.; Yasumoto, T.; Shibayama, T.; Ohtani, B. Decahedral Single-Crystalline Particles of Anatase Titanium (IV) Oxide with High Photocatalytic Activity. Chem. Mater. 2009, 21, 2601-2603. [CrossRef]

12. Ye, L.Q.; Liu, J.Y.; Tian, L.H.; Peng, T.Y.; Zan, L. The replacement of $\{101\}$ by $\{010\}$ facets inhibits the photocatalytic activity of anatase $\mathrm{TiO}_{2}$. Appl. Catal. B. Environ. 2013, 134, 60-65. [CrossRef]

13. Zheng, Z.; Huang, B.; Lu, J.; Qin, X.; Zhang, X.; Dai, Y. Hierarchical TiO 2 Microspheres: Synergetic Effect of $\{001\}$ and $\{101\}$ Facets for Enhanced Photocatalytic Activity. Chem. Eur. J. 2011, 17, 15032-15038. [CrossRef] [PubMed]

14. Meng, A.; Zhang, J.; Xu, D.; Cheng, B.; Yu, J. Enhanced photocatalytic $\mathrm{H}_{2}$-production activity of anatase $\mathrm{TiO}_{2}$ nanosheet by selectively depositing dual-cocatalysts on $\{101\}$ and $\{001\}$ facets. Appl. Catal. B. Environ. 2016, 198, 286-294. [CrossRef]

15. Sun, D.; Yang, W.; Zhou, L.; Sun, W.; Li, Q.; Shang, J.K. The selective deposition of silver nanoparticles onto $\{101\}$ facets of $\mathrm{TiO}_{2}$ nanocrystals with co-exposed $\{001\} /\{101\}$ facets, and their enhanced photocatalytic reduction of aqueous nitrate under simulated solar illumination. Appl. Catal. B. Environ. 2016, 182, 85-93. [CrossRef]

16. Murakami, N.; Kurihara, Y.; Tsubota, T.; Ohno, T. Shape-Controlled Anatase Titanium (IV) Oxide Particles Prepared by Hydrothermal Treatment of Peroxo Titanic Acid in the Presence of Polyvinyl Alcohol. J. Phys. Chem. C 2009, 113, 3062-3069. [CrossRef]

17. Chamtouri, M.; Kenens, B.; Aubert, R.; Lu, G.; Inose, T.; Fujita, Y.; Masuhara, A.; Hofkens, J.; Uji-i, H. Facet-Dependent Diol-Induced Density of States of Anatase $\mathrm{TiO}_{2}$ Crystal Surface. ACS Omega 2017, 2, 4032-4038. [CrossRef] [PubMed]

18. Liu, C.; Han, X.; Xie, S.; Kuang, Q.; Wang, X.; Jin, M.; Xie, Z.; Zheng, L. Enhancing the Photocatalytic Activity of Anatase $\mathrm{TiO}_{2}$ by Improving the Specific Facet-Induced Spontaneous Separation of Photogenerated Electrons and Holes. Chem. Asian. J. 2012, 8, 282-289. [CrossRef] [PubMed] 
19. Wang, W.; Lai, M.; Fang, J.; Lu, C. Au and Pt selectively deposited on $\{001\}$-faceted $\mathrm{TiO}_{2}$ toward SPR enhanced photocatalytic $\mathrm{Cr}(\mathrm{VI})$ reduction: The influence of excitation wavelength. Appl. Surf. Sci. 2018, 439, 430-438. [CrossRef]

20. Murakami, N.; Kawakami, S.; Tsubota, T.; Ohno, T. Dependence of photocatalytic activity on particle size of a shape-controlled anatase titanium (IV) oxide nanocrystal. J. Mol. Catal. A. Chem. 2012, 358, $106-111$. [CrossRef]

21. Xiong, Z.; Lei, Z.; Chen, X.; Gong, B.; Zhao, Y.; Zhang, J.; Zheng, C.; Wu, J.C.S. CO 2 photocatalytic reduction over Pt deposited $\mathrm{TiO}_{2}$ nanocrystals with coexposed $\{101\}$ and $\{001\}$ facets: Effect of deposition method and Pt precursors. Catal. Commun. 2017, 96, 1-5. [CrossRef]

22. Jiang, Z.; Ding, D.; Wang, L.; Zhang, Y.; Zan, L. Interfacial effects of $\mathrm{MnOx}-$ loaded $\mathrm{TiO}_{2}$ with exposed $\{001\}$ facets and its catalytic activity for the photoreduction of $\mathrm{CO}_{2}$. Catal. Sci. Technol. 2017, 7, 3065-3072. [CrossRef]

23. Ohno, T.; Sarukawa, K.; Matsumura, M. Crystal faces of rutile and anatase $\mathrm{TiO}_{2}$ particles and their roles in photocatalytic reactions. New. J. Chem. 2002, 26, 1167-1170. [CrossRef]

24. Li, R.; Zhang, F.; Wang, D.; Yang, J.; Li, M.; Zhu, J.; Zhou, X.; Han, H.; Li, C. Spatial separation of photogenerated electrons and holes among $\{010\}$ and $\{110\}$ crystal facets of $\mathrm{BiVO}_{4}$. Nat. Commun. 2013, 4, 1432. [CrossRef] [PubMed]

25. Zhou, C.; Wang, S.; Zhao, Z.; Shi, Z.; Yan, S.; Zou, Z. A Facet-Dependent Schottky-Junction Electron Shuttle in a $\mathrm{BiVO}_{4}\{010\}-\mathrm{Au}-\mathrm{Cu}_{2} \mathrm{O} Z-S c h e m e$ Photocatalyst for Efficient Charge Separation. Adv. Funct. Mater. 2018, 28, 1801214. [CrossRef]

26. Guo, Y.; Siretanu, I.; Zhang, Y.; Mei, B.; Li, X.; Mugele, F.; Huang, H.; Mul, G. pH-Dependence in facet-selective photo-deposition of metals and metal oxides on semiconductor particles. J. Mater. Chem. A 2018, 6, 7500-7508. [CrossRef]

27. Zhang, Q.; Li, R.; Li, Z.; Li, A.; Wang, S.; Liang, Z.; Liao, S.; Li, C. The dependence of photocatalytic activity on the selective and nonselective deposition of noble metal cocatalysts on the facets of rutile $\mathrm{TiO}_{2}$. J. Catal. 2016, 337, 36-44. [CrossRef]

28. Wenderich, K.; Klaassen, A.; Siretanu, I.; Mugele, F.; Mul, G. Sorption-Determined Deposition of Platinum on Well-Defined Platelike $\mathrm{WO}_{3}$. Angew. Chem. Int. Edit. 2014, 53, 12476-12479. [CrossRef]

29. Ohtani, B.; Bowman, R.M.; Colombo, D.P., Jr.; Kominami, H.; Noguchi, H.; Uosaki, K. Femtosecond Diffuse Reflectance Spectroscopy of Aqueous Titanium (IV) Oxide Suspension: Correlation of Electron-Hole Recombination Kinetics with Photocatalytic Activity. Chem. Lett. 1998, 27, 579-580. [CrossRef]

30. Lazzeri, M.; Vittadini, A.; Selloni, A. Structure and energetics of stoichiometric $\mathrm{TiO}_{2}$ anatase surfaces. Phys. Rev. B 2001, 63, 155409. [CrossRef]

31. Lazzeri, M.; Vittadini, A.; Selloni, A. Erratum: Structure and energetics of stoichiometric $\mathrm{TiO}_{2}$ anatase surfaces. Phys. Rev. B 2002, 65, 119901. [CrossRef]

32. Janczarek, M.; Kowalska, E.; Ohtani, B. Decahedral-shaped anatase titania photocatalyst particles: Synthesis in a newly developed coaxial-flow gas-phase reactor. Chem. Eng. J. 2016, 289, 502-512. [CrossRef]

33. Wei, Z.; Kowalska, E.; Ohtani, B. Influence of Post-Treatment Operations on Structural Properties and Photocatalytic Activity of Octahedral Anatase Titania Particles Prepared by an Ultrasonication-Hydrothermal Reaction. Molecules 2014, 19, 19573-19587. [CrossRef] [PubMed]

34. Kajita, M.; Kuwabara, T.; Hasegawa, D.; Yagi, M. Element-saving preparation of an efficient electrode catalyst based on self-assembly of Pt colloid nanoparticles onto an ITO electrode. Green Chem. 2010, 12, $2150-2152$. [CrossRef]

35. Frens, G. Controlled Nucleation for Regulation of Particle-Size in Monodisperse Gold Suspensions. Nature 1973, 20-22. [CrossRef]

(C) 2018 by the authors. Licensee MDPI, Basel, Switzerland. This article is an open access article distributed under the terms and conditions of the Creative Commons Attribution (CC BY) license (http://creativecommons.org/licenses/by/4.0/). 